Gefässchirurgie 2013 · 18:12-13

DOI 10.1007/s00772-012-1105-5

Online publiziert: 13. Februar 2013

(c) Springer-Verlag Berlin Heidelberg 2013
H. Wenk

Klinik für Chirurgie und Gefäßzentrum Bremen-Nord, Klinikum Bremen Nord

\section{Leitlinien für} perioperative Medizin?
Seit 5 Jahren gibt es eine Kommission „Perioperative Medizin“ in der Deutschen Gesellschaft für Gefäßchirurgie und Gefäßmedizin (DGG, • Infobox 1). Die Kongresse der DGG in Berlin (Debus 2010), Erlangen (Lang 2011) und Wiesbaden (Schmitz-Rixen 2012) boten Schwerpunktthemen der perioperativen Medizin, die auf erfreulich positive Resonanz stießen.

Offensichtlich besteht an einer Diskussion und Wissensvermittlung Bedarf, was präoperativ an Diagnostik sinnvoll und erforderlich ist, wie eine operative oder interventionelle Behandlung geplant werden kann, welche Medikamente an- und abgesetzt werden müssen und wie die postoperative Therapie und Nachsorge gestaltet werden sollte.

Diese Nachfrage scheint darin begründet, dass sich bei der Patientenbehandlung nicht nur durch die zunehmende Spezialisierung der chirurgischen Fächer ein Paradigmenwechsel vollzieht. Dies gilt nicht nur für die Behandlung von Krankheitsbildern, sondern insbesondere auch für das Erlernen des Umgangs mit dem $\mathrm{Pa}$ tienten.

\section{Entwicklung der Medizin von einer ganzheitlichen Betrachtungsweise zur Behandlung von Krankheitsbildern}

Nur eine Medizinergeneration zurück wurden Patienten nach ihrer Aufnahme in einer Krankeneinrichtung komplett untersucht, ein breites Spektrum von Laborwerten ("große Laborlatte") bestimmt und obligat auf das Vorliegen von Geschlechtskrankheiten hin untersucht (Screening!). Zudem wurde von jedem Patienten ein
Elektrokardiogramm (EKG) erstellt und eine Röntgenuntersuchung des Thorax angefertigt. In Abhängigkeit von den Untersuchungsergebnissen wurde seine Behandlung festgelegt. Fand man bei einer Varikose ein Ulcus ventriculi, wurde zunächst das Ulkus behandelt, fand man bei einem Aortenaneurysma eine symptomatische Karotisstenose, wurde zuerst die Halsschlagader operiert.

Mit der fortschreitenden Spezialisierung der chirurgischen Fächer, vor allem aber mit der Einführung des pauschalierten Entgeltsystems (DRG), trat dieser ganzheitliche Behandlungsansatz in den Hintergrund.

Heute behandeln wir nicht mehr einen Patienten, sondern ein Krankheitsbild. Die ganzheitliche Sichtweise auf den Patienten ist einer Auftragsleistung gewichen. Es wird die Erkrankung behandelt, wegen der ein Patient vom Zuweiser eingewiesen wurde. Und genau diese Behandlung wird vom Kostenträger vergütet.

Konsekutiv wird aller Ballast abgeworfen, der nicht oder nicht unmittelbar etwas mit diesem Krankheitsbild zu tun hat. Bei Koinzidenz zweier Krankheitsbilder wird tatsächlich nur eines behandelt, der Patient entlassen und anschließend (ggf. mit einer zweiten Diagnose) als „neuer Fall“ wieder aufgenommen.

Das Abspecken an Diagnostik kann für den Patienten durchaus komfortabel sein. Doppeluntersuchungen werden reduziert, die Überlegungen, welche Untersuchungen tatsächlich sinnvoll, zielführend und evidenzbasiert sind, können hilfreich sein und dienen darüber hinaus der Kostenreduzierung im Gesundheitssystem. Sie können aber auch ein kritisches Mindestmaß unterschreiten, was das Behand- lungsrisiko steigert und damit die Behandlungsqualität gefährdet.

\section{Leitlinien und Behandlungsqualität}

Leitlinien können dabei helfen, die Behandlungsqualität zu sichern. Sie tun das am besten dann, wenn sie sich an einer konkreten Krankheitsentität orientieren. Die von der Deutschen Gesellschaft für Gefäßchirurgie veröffentlichten Leitlinien zu Diagnostik und Therapie in der Gefäßchirurgie sind diesbezüglich nur bedingt tauglich [1]. So nimmt beispielsweise die S2-Leitlinie zu den Bauchaorten- und Beckenarterienverschlüssen in diesem Werk zur Basisdiagnostik und zur weiterführenden Diagnostik relativ dezidiert Stellung.

Einen Quantensprung zeigt dagegen die aktuelle S3-Leitlinie zur Behandlung der Karotisstenose. Ob die S3-Leitlinie tatsächlich eine Handlungshilfe im klinischen Alltag sein kann, darf allein wegen ihres Umfangs hinterfragt werden [2]. Sie ist aber ein umfassendes Werk, die das gesamte komplexe Gebiet der Karotisstenosen und ihrer endovaskulären und offen operativen Therapie unter den Aspekten der evidenzbasierten Medizin abbildet und erklärt. Der Diagnostik von Karotisstenosen ist dabei ein eigenes Kapi-

\section{Infobox 1 Kommission "Perioperative Medizin"}

Johannes Gahlen (Klinikum Ludwigsburg) Jörg Heckenkamp (Gefäßzentrum Osna brück) Monique Jakobs (DGG, Berlin) Knut Walluscheck (Gefäßzentrum Flensburg) Eike Weißflog (Klinikum-Bremen-Nord) Heiner Wenk (Gefäßzentrum Bremen-Nord) Birgit Wulff (Universitätsklinik Münster) 
tel gewidmet [6]. Unbeachtet des großen Umfangs der Leitlinie erhält der Arzt und Praktiker klare, abgesicherte und untermauerte Informationen zu einer speziellen Erkrankung.

\section{》) Leitlinien vermitteln \\ dem Arzt klare und abgesicherte Informationen zu speziellen Erkrankungen}

Weniger zielführend können dabei allgemeine Publikationen wie „perioperative Risikoabklärung aus kardiologischer Sicht sein“ [4], da damit ja wieder die ganzheitliche, fachbezogen internistische Sichtweise über die erkrankungsbezogene Darstellung evidenzbasierter Handlungsanweisungen für spezielle gefäßchirurgische Krankheitsbilder dominiert.

\section{Arbeitsschwerpunkte der Kommission „Perioperative Medizin"}

Die Kommission „Perioperative Medizin“ der Deutschen Gesellschaft für Gefäßchirurgie hat sich bisher vorwiegend mit den perioperativen Aspekten des Aortenpatienten beschäftigt. Dazu gehörten folgende Themen:

- Umsetzung des Fast-track-Konzepts bei endovaskulären und offenen Operationen (Heckenkamp/Wenk)

- Periduralanästhesie bei Aortenaneurysmaoperation (Heckenkamp/ Wulff)

- Perioperatives Gerinnungsmanagement (Wulff)

- Aufklärung/Operationsbericht (Walluscheck/Wenk)

- Klinische Pfade (Walluscheck/Gahlen)

Am Krankheitsbild „Aortenaneurysma“ wurden die Schnittstellenprobleme zwischen Hausärzten, Anästhesie, Intensivmedizin und den verschiedenen chirurgischen Disziplinen dargelegt [8]. Über die perioperative Medikation bei arteriellen Operationen wurde berichtet [5]. Es kann festgehalten werden, dass eine $\beta$-BlockerTherapie beibehalten werden sollte, dass Statine mindestens einen Monat präoperativ und einen Monat postoperativ gege- ben werden sollten. Thrombozytenaggregationshemmer sollten perioperativ nicht pausiert werden, es sei denn, es besteht ein deutlich erhöhtes Blutungsrisiko. Eine duale Plättchenaggregationshemmung sollte aber pausiert werden. Nach Koronarintervention muss ggf. ein entsprechendes Zeitintervall eingehalten werden.

Das Aortenaneurysma und seine verschiedenen Behandlungsmöglichkeiten (endovaskulär, offen, „Hybrid“) ist beispielhaft für die Übernahme der viszeralchirurgisch definierten Behandlungskonzepte des „fast track“ in die Gefäßmedizin. Die endovaskuläre Behandlung des Aortenaneurysmas ist per se eine Fast-trackProzedur. Debus et al. haben bereits 2009 über die Aspekte der Fast-track-Medizin in der Gefäßchirurgie berichtet.

Die Anästhesiemethoden als Voraussetzung für die Behandlung des Aortenaneurysmas finden im Moment besondere Aufmerksamkeit, zumal der Periduralkatheter eine Renaissance erlebt und seine Anwendung im Zeitalter der Thrombozytenaggregationshemmer besonders differenziert indiziert werden muss. Die Aufklärung und das Abfassen von Operationsberichten war im Rahmen der letzten beiden Jahreskongresse Thema von „Pround Kontra-Sitzungen“.

Mit der Ökonomisierung wurde aus der Medizin als Mittel zum Zweck die Medizin zum Selbstzweck [7]. Die ganzheitliche Medizin, die auf den Pathologen Rudolph Virchow zurückgeht, bei der ein politisches und wissenschaftliches Weltbild eins wird, weicht einer Organ- oder Systemmedizin. Dies ist für die perioperative Medizin eine Herausforderung. Mit der Entwicklung weg von der ganzheitlichen Betrachtung des Menschen und des Patienten könnte das Wohl des Patienten zugunsten eines optimalen Behandlungsergebnisses für das behandelte Organ(system) verloren gehen.

Die perioperative Medizin ist in diesem Zusammenhang ein Korrektiv. Sie überlegt, wie ein Eingriff, eine Operation zum Wohl und zur Genesung des Patienten in einen Behandlungsalgorithmus sinnvoll integriert werden kann und welcher Eingriff für welchen Patienten zu einem sinnvollen Ergebnis führt.
Sie braucht neben einer Qualitätskontrolle Handlungsanweisungen, die in Form von Leitlinien den aktuellen Wissensstand wiedergeben und Handlungskorridore in dezidierten Situationen anbieten.

Die Ökonomisierung und die Evidenzbasierung der Medizin sind dabei nicht zum Nulltarif zu haben. Wer die Kosten für die Erstellung einer S3-Leitlinie kennt, dem kommen Zweifel auf, ob die Medizin durch diese Entwicklung preiswerter wird. Einen Weg zurück zur eminenzbasierten Medizin wird es nicht geben. So bleibt nur, mit der perioperativen Medizin diese Einbahnstraße sinnvoll auszuschildern.

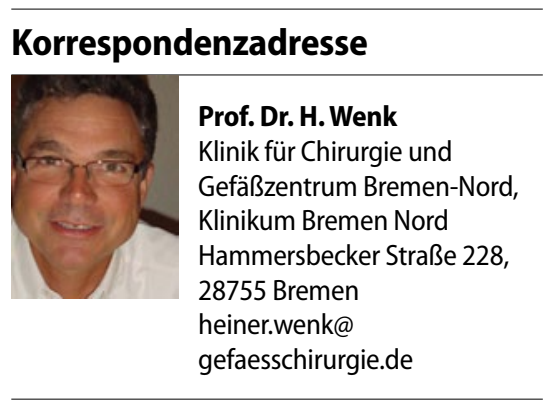

\section{Literatur}

1. Deutsche Gesellschaft für Gefäßchirurgie (2010) Leitlinien zu Diagnostik und Therapie in der Gefäßchirurgie. Springer, Berlin Heidelberg New York Tokio

2. Eckstein HH et al. (2012) S3-Leitlinie zur Diagnostik, Therapie und Nachsorge der extracraniellen Carotisstenose. Gefässchirurgie 17: 495-496

3. Debus, ES, Kruska P, Ivoghli A et al. (2009) Fast Track in der Gefäßchirurgie. Chirurg 80, 711-718

4. Markworth F, Debus ES, Blankenberg S (2013) Elektive nicht kardiochirurgische Operationen bei Patienten mit kardialer Grunderkrankung. Präopertive Risikoeinschätzung und perioperatives Vorgehen. Gefässchirurgie 18(1): 21-27

5. Nowak T, Weißflog E, Böhner H, Wenk H (2011) Perioperative Medikation bei arteriellen Operationen. Was ansetzen und was absetzen? Gefässchirurgie 16(5):324-329

6. Ringleb P, Görtler M, Nabavi DG et al. (2012) Symptome und Diagnostik von Carotisstenosen. Gefässchirurgie 17: 502-519

7. Unschudt P (2009) Ware Gesundheit. CH Beck, München

8. Wenk HH, Weißflog E, Wulff B et al. (2011) Schnittstellenprobleme und -lösungen bei der elektiven Behandlung des Bauchaortenaneurysmas. Gefässchirurgie16(3):192-198 